\title{
Common and divergent functions of Beclin 1 and Beclin 2
}

\author{
Cell Research (2013) 23:1341-1342. doi:10.1038/cr.2013.129; published online 10 September 2013
}

In a recent paper published in Cell, He and colleagues reported the identification and functional characterization of Beclin 2, a mammalspecific homolog of the evolutionarily conserved autophagy-regulatory and oncosuppressive factor Beclin 1. In spite of a non-negligible degree of sequence identity, Beclin 1 and Beclin 2 differ from each other in multiple aspects, including their functional profile as well as the genomic organization of the respective loci.

Originally identified as a BCL-2-interacting partner capable of protecting mice from viral encephalitis [1], Beclin 1 - the mammalian ortholog of yeast Atg6 - is nowadays well known as a core component of the class III phosphoinosite-3-kinase (PI3K) enzymatic complex that initiates the formation of autophagosomes in the course of macroautophagy (hereafter referred to as autophagy) [2]. Presumably owing to the critical function of autophagy in embryonic development, mice lacking both copies of the Beclin 1-coding gene (Becn 1) die early during embryogenesis. Moreover, Becn $1^{+/-}$mice suffer from a high incidence of spontaneous tumors, indicating that Beclin 1 acts as a haploinsufficient tumor suppressor [3]. At least in part, this reflects the central role that autophagy plays in the maintenance of intracellular homeostasis. Indeed, baseline levels of autophagy mediate the removal of various cytoplasmic entities that might favor oncogenesis, including damaged mitochondria and protein aggregates [4]. Conversely, established neoplasms often harness the cytoprotective functions of autophagy to their own benefit
[2]. The pathophysiological relevance of autophagy is not limited to cancer, but extends to a large panel of human diseases, including neurodegenerative, cardiovascular and infectious conditions [5]. Thus, during the last decade autophagy-regulatory signaling pathways have been intensively investigated.

Until now, Beclin 1 was considered as the only Beclin encoded by the mammalian genome, sharing some degree of structural homology with so-called "BH3-only" proteins, pro-apoptotic members of the BCL-2 family that are involved in the activation of cell death in response to stress [6]. In a recent paper published in Cell, the research group led by Beth Levine [7] identified a human and a mouse protein sharing $57 \%$ and $44 \%$ sequence identity with human and mouse Beclin 1, respectively, de facto unveiling the existence of an additional, mammal-specific ortholog of Atg6, Beclin 2. The mouse Beclin 2 mRNA was detected in multiple organs including the brain, skeletal muscle, placenta, thymus and uterus, as was the human protein in both fetal and adult brain tissues. These data demonstrate that the current classification of mouse and human Beclin 2-encoding genes (i.e., NG_022940 and NG_028451) as pseudogenes is incorrect.

The knockdown of Beclin 2 reduced several manifestations of basal or starvation-induced autophagy in cultured mammalian cells, including the degradation of the autophagic substrate p62, the aggregation of a fluorescent form of LC3 into cytoplasmic dots and the lipidation of endogenous LC3. All such effects, which were not due to an increased autophagosomal turnover (as verified in the presence of the lysosomal inhibitor bafilomycin A1), could be rescued upon the transgene-driven expression of a non-interferable Beclin 2 variant. Thus, similar to Beclin 1, Beclin 2 regulates autophagy [7]. In fact, Beclin 2 turned out to physically interact with several (but not all) components of the class III PI3K complex organized around Beclin 1, including the catalytic subunit VPS34 as well as the regulatory factors ATG14, AMBRA1 and UVRAG, but not RUBICON (Figure 1A). Beclin 2 also appeared to share with Beclin 1 the ability to bind BCL-2, although only the latter gets dissociated from such an interaction in the course of stress-induced autophagy [7, 8]. As the greatest divergence between mammalian Beclins involves their $\mathrm{N}$ terminus, He and colleagues employed the N-terminal domain of Beclin 2 as a bait in a yeast two-hybrid screen, and identified $G$ protein-coupled receptor (GPCR)-associated sorting protein 1 (GASP1) as a Beclin 2-specific interactor. Thus, similar to GASP1 (but not to Beclin 1), Beclin 2 was required for the agonist-induced lysosomal degradation of a subset of GPCRs including opioid receptor $\delta 1$ (DOR) and cannabinoid receptor 1 (CB1R). Importantly, such an activity, but not the capacity of Beclin 2 to regulate autophagic responses, appears to rely on the physical interaction between Beclin 2 and GASP1.

To obtain insights into the physiological functions of Beclin 2, He and colleagues attempted to generate $B e c n 2^{--}$mice, finding that these animals survived embryonic and early post-natal development at sub-Mendelian rates (approximately $4 \%$ ). Not only 

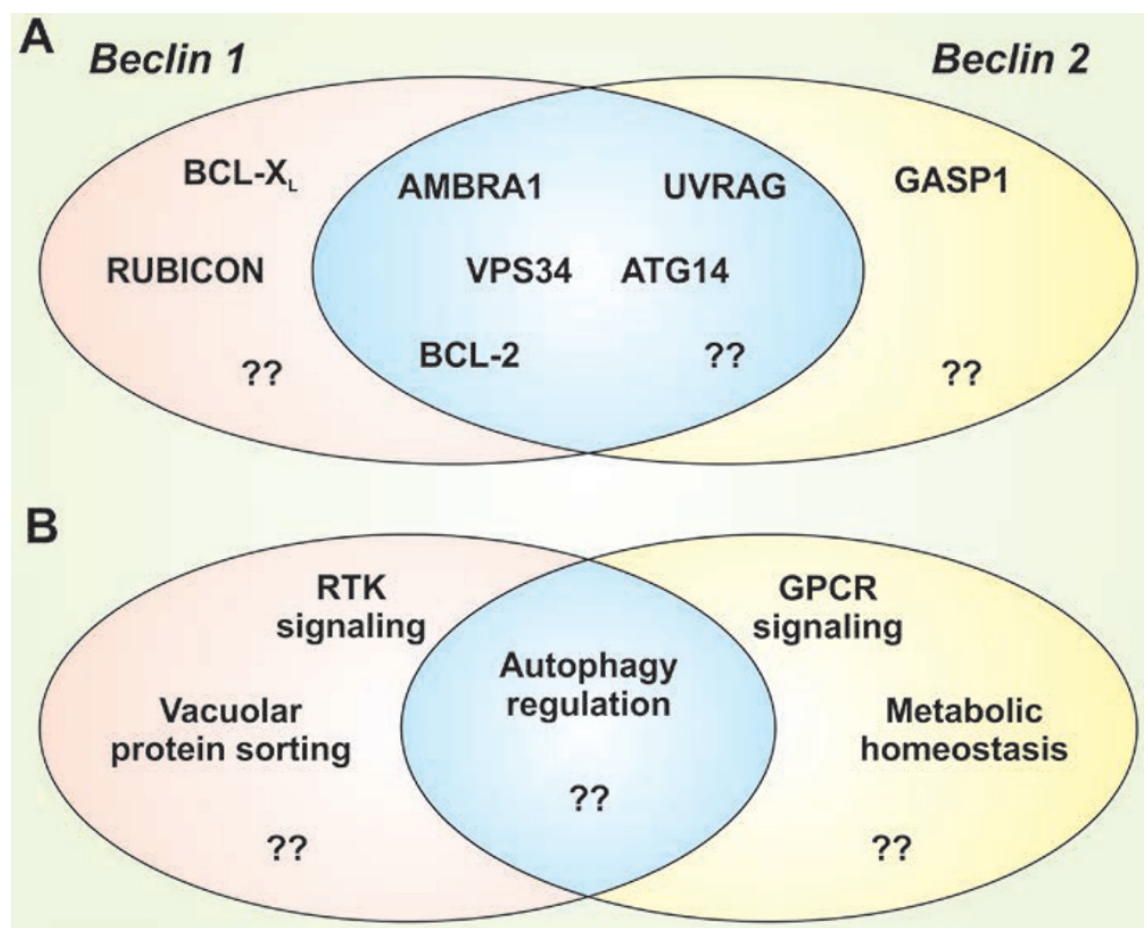

Figure 1 Common and divergent functions of mammalian Beclins. Specificity of the main interactors (A) and functions (B) ascribed to mammalian Beclin 1 and Beclin 2 to date. GPCR, G protein-coupled receptor; RTK, receptor tyrosine kinase.

$B e c n 2^{+/-}$and Becn2 $2^{-/-}$mouse embryonic fibroblasts, but also the brain of Becn2 $^{+/-}$animals exhibited significant autophagic defects, corroborating the role of Beclin 2 in the regulation of autophagy in vivo. Moreover, these genotypes were associated with increased basal levels of multiple GPCRs, including $\mathrm{CB} 1 \mathrm{R}$ and dopamine receptor $\mathrm{D} 2$ (DRD2) [7]. In line with the notion that increased CB1R signaling accrues food intake and hence favors obesity and insulin resistance, while pharmacological or genetic CB1R inhibition has opposite effects [9], Becn2 ${ }^{+/-}$mice accumulated more weight than their wild-type littermates in response to a standard (as well as to a high-fat) diet. At odds with their Becn $1^{+/}$counterparts, Becn2 $2^{+/-}$mice also exhibited impaired glucose tolerance and decreased insulin sensitivity, two effects that could be reverted by a chemical CB1R antagonist [7]. Taken together, these data demonstrate that

Beclin 1 is known to regulate various processes other than autophagy, including vacuolar protein sorting and the degradation of specific growth factor receptors [10]. Thus, in spite of $44 \%-57 \%$ sequence identity, the two mammalian Beclins described to date are relatively different from each other, exhibiting functional profiles that overlap to a limited degree (Figure 1B). Interestingly, $\mathrm{He}$ and colleagues have previously shown that defects in stimulus-induced autophagy (including those introduced by the BecnI $1^{+/-}$genotype) are coupled to decreased endurance and altered glucose metabolism during acute exercise, training to protect mice against dietinduced glucose intolerance [8]. Part of these phenomena were shown to reflect defects in the AMP-activated protein kinase (AMPK)-dependent exposure of glucose transporters on the plasma membrane of skeletal muscle cells. It is therefore tempting to speculate that the metabolic phenotype of Becn $2^{+/-}$may in part originate from peripheral defects in glucose handling linked to autophagy. Thus, although the force driving the divergence of mammalian Beclins remains to be elucidated, it may reflect the need for an integrated regulation of central and peripheral mechanisms of metabolic homeostasis. Further studies are required to address this hypothesis.

Lorenzo Galluzzi ${ }^{1,2,3}$,
Guido Kroemer',3,4,5,6

${ }^{I}$ Gustave Roussy, Villejuif, France; ${ }^{2}$ Université Paris Descartes/Paris V, Sorbonne Paris Cité, Paris, France; ${ }^{3}$ Equipe 11 labellisée Ligue Nationale contre le Cancer, Centre de Recherche des Cordeliers, Paris, France; ${ }^{4}$ INSERM, U848, Villejuif, France; 'SPôle de Biologie, Hôpital Européen Georges Pompidou, AP-HP, Paris, France; ${ }^{6}$ Metabolomics and Cell Biology Platforms, Gustave Roussy, Villejuif, France Correspondence: Guido Kroemera ${ }^{\mathrm{a}}$,

Lorenzo Galluzzi ${ }^{\mathrm{b}}$

${ }^{a}$ Tel: +33 14211 4516; Fax: +33 142116047

E-mail: kroemer@orange.fr

'Tel: +33 14211 6046; Fax: +33 142116047

E-mail: deadoc@vodafone.it as well as with an impaired capacity of

\section{References}

1 Liang XH, Kleeman LK, Jiang HH, et al. J Virol 1998; 72:8586-8596.

2 Kroemer G, Marino G, Levine B. Mol Cell 2010; 40:280-293.

3 Yue Z, Jin S, Yang C, et al. Proc Natl Acad Sci USA 2003; 100:15077-15082.

4 Green DR, Galluzzi L, Kroemer G. Science 2011; 333:1109-1112.

5 Choi AM, Ryter SW, Levine B. $N$ Engl J Med 2013; 368:651-662.

6 Sinha S, Levine B. Oncogene 2008; 27:S137-S148.

7 He C, Wei Y, Sun K, et al. Cell 2013;154:1085-1099.

8 He C, Bassik MC, Moresi V, et al. Nature 2012; 481:511-515.

9 Maccarrone M, Gasperi V, Catani MV, et al. Annu Rev Nutr 2010; 30:423-440.

10 Funderburk SF, Wang QJ, Yue Z. Trends Cell Biol 2010; 20:355-362. 